\title{
Re-thinking treatment strategies for febrile neutropenia in paediatric oncology population: $A$ perspective from a developing country.
}

Vinson James ( $\nabla$ dr.vinsonjames@gmail.com )

Assumption Hospital https://orcid.org/0000-0003-2897-925X

Anand Prakash

St Johns Medical College Hospital

Kayur Mehta

Montreal Children's Hospital Glen Site McConnell Resource Centre Medical Library

Tarangini Durugappa

St Johns Medical College Hospital

Research article

Keywords: febrile neutropenia, clinical profile, antibiotic sensitivity, microbiological profile

Posted Date: April 22nd, 2020

DOI: https://doi.org/10.21203/rs.3.rs-23053/v1

License: (a) (i) This work is licensed under a Creative Commons Attribution 4.0 International License.

Read Full License

Version of Record: A version of this preprint was published at Infectious Agents and Cancer on June 19th, 2021. See the published version at https://doi.org/10.1186/s13027-021-00387-y. 


\section{Abstract}

Background: This study was conducted to evaluate the microbiological profile of bacterial isolates in febrile neutropenia in a pediatric oncology unit, thereby, reviewing the use of restricted antibiotics and need for aggressive medical treatment accordingly.

Methods: A prospective observational study was conducted in a paediatric haemat-oncology division of a tertiary care teaching hospital in southern India from September 2014 to August 2016. Children with febrile neutropenia were enrolled in the study. Blood cultures were performed using automated system. Cultures from other sites were obtained if needed, based on the clinical profile. Standard antibiotic susceptibility testing was done. Statistical analysis was done using SPSS.

Results: One hundred and thirty children were enrolled for the study. Two hundred and fifty episodes of febrile neutropenia were studied. Three hundred and eighty four cultures were sent and $92(24 \%)$ cultures were positive. There were $52.2 \%$ gram negative isolates followed $35.8 \%$ gram positive isolates, $6.5 \%$ fungal isolates and $5.5 \%$ poly-microbial cultures. Lactose fermenting gram negative bacteria ( 29 isolates, $31.5 \%$ ) were the most frequently isolated in the gram negative group, with Escherichia coli being the most common organism (19 isolates, $20.6 \%$ ). Amongst gram positive coagulase negative staphylococcus (CONS) was the most common (29\%). Escherichia coli and NFGNB had only $36 \%, 25 \%$ sensitivity to ceftazidime respectively. Most gram negative bacteria were found to have better sensitivity to amikacin (mean: 57). There was a higher prevalence of extended spectrum beta lactamase producing organisms. 36 out of $48 \mathrm{GNB}$ were found to be either multi/extremely/pan drug resistant. Escherichia coli and Klebsiella were often drug resistant. Significantly higher mortality was associated with gram negative isolates $(61.5 \%)$

Conclusions: In view of higher prevalence of gram negative isolates and emergence of multi drug resistance, frequent audits of resistance patterns should guide the choice of antimicrobials in febrile neutropenia management. Our results show the importance of surveillance, monitoring resistance frequencies and identifying risk factors specific to each region. Given that significant mortality is attributed to drug resistant gram negative bacteria, early initiation of appropriate antibiotics to cover for drug resistance is required while formulating empirical antibiotic policies for febrile neutropenia in the oncology units in the developing world.

\section{Introduction}

Keeping abreast of the changes in spectrum of bacterial infections and trends in drug resistance is essential in paediatric oncology units. Intensive chemotherapy in leukemia and other malignancies causes neutropenia and febrile episodes. [1] Neutropenia is defined as an Absolute Neutrophil Count (ANC) $<500 / \mu \mathrm{L}$ or less than $1000 / \mu \mathrm{L}$ with an anticipated decline to less than $500 / \mu \mathrm{L}$ in the next 48 -hour period. Neutropenic fever is a single oral temperature of $38.3^{\circ} \mathrm{C}\left(101^{\circ} \mathrm{F}\right)$ or a temperature of greater than $38.0^{\circ} \mathrm{C}\left(100.4^{\circ} \mathrm{F}\right)$ sustained for more than one hour in a patient with neutropenia. Although febrile 
neutropenia can be non-infectious, most are caused by infectious agents. [2] Empirical antibiotic therapy (broad spectrum antibiotic) has been successfully used in the management of febrile neutropenia.

Recent trends in febrile neutropenia in children suggest an emergence of uncommon and drug resistant gram negative bacteria as the most important cause of morbidity and mortality.[3] Such infections are common in immunocompromised conditions especially malignancies. [4]There is variability in drug resistance patterns among various isolates. [5] So, appropriate empirical antibiotics need to be chosen carefully. Gram positive isolates may have drug resistance commonly methicillin resistance. Many studies show that patients with gram negative infections have poorer prognosis and higher mortality compared to gram-positive bacteraemia. [6] Due to higher prevalence of drug resistance and mortality in gram negative organisms, the treatment regimen chosen for empirical antibiotics is of paramount importance. The changing microbiological spectrum, in febrile neutropenia and the resistance patterns, help guide antibiotic treatment. [7]

Audit of febrile neutropenic episodes, would help guide treatment protocols to reduce the mortality and improve outcomes. We conducted a single centre prospective study aimed at identifying the trends in the pattern of microbiological isolates and sensitivity patterns for episodes of febrile neutropenia in the paediatric oncology unit of a tertiary care teaching hospital. The trends that were revealed can help guide optimal antimicrobial therapy.

\section{Materials And Methods}

A prospective observational study was conducted at the Division of Paediatric Hemat-Oncology from 1st September 2014 to 31st August 2016. Neutropenia was defined as Absolute Neutrophil Count (ANC) $<500 / \mu \mathrm{L}$ or less than $1000 / \mu \mathrm{L}$ with an anticipated decline to less than $500 / \mu \mathrm{L}$ in the next 48 -hour period. The criterion for inclusion into the study was children (i.e., less than 18 years) with various malignancies diagnosed with febrile neutropenia. Those with fever, that occurred during the administration of chemotherapy (i.e., less than 24 hours) or fever occurring during or within six hours of transfusion of blood or blood products were excluded. A detailed history regarding fever, type of cancer, chemotherapy regimen, was recorded and physical examination was conducted.

Blood counts and blood cultures were drawn and first line intravenous antibiotics (Ceftazidime and amikacin) were started as per unit protocol, in paediatric emergency room. If a child had a central venous access device, blood cultures were drawn only from that device. Urine culture was done in patients with urinary symptoms Endotracheal tube trap culture was sent of patients intubated during the course of hospitalisation where clinically indicated. Cerebrospinal fluid culture was sent in those who developed neurological symptoms during the course of febrile neutropenia. Identification of the pathogens was done by automated technique for blood and manual plate culture technique for urine, cerebrospinal fluid and endotracheal tube trap collection. Standard testing by plate method was done to find the antibiotic susceptibility for suspected urinary tract infection (UTI). Shock was treated as per the Surviving sepsis guidelines and such patients were monitored in the Intensive Care Unit (ICU). [8, 9] Antibiotics were 
stopped once the patient was afebrile for 48 hours with if ANC $>500 / \mathrm{mm}^{3}$ for two consecutive days with no definite site of infection and a negative culture report.

Change to higher antibiotics (second/third line antimicrobials) was considered on non-resolution of fever/ signs of persistent active infections (like rigors or hemodynamic instability) and based on results of culture/sensitivity reports.

Ceftazidime and amikacin were prescribed as first line antibiotics. In episodes of persisting fever, second line antibiotic (piperacillin/ tazobactam and amikacin) was started. If there was persistent fever with hemodynamic compromise, third line drug (meropenem and vancomycin) was initiated till initial blood culture reports were available. Amphotericin B was the anti-fungal of choice. Voriconazole was used for Aspergillus infections. Dengue haemorrhagic fever was suspected if patient presented with signs of tender hepatomegaly, third spacing of fluids and was positive based on dengue serology. Dengue fever was treated based on standard guidelines. [10]

The restricted antibiotics for use in this study were colistin, meropenem, vancomycin and tigecycline. Sensitivity patterns were analysed and resistance patterns were mapped. Multi-drug resistance (MDR) was defined as acquired non-susceptibility to at least one agent in three or more antimicrobial categories. Extensive drug resistance (XDR) was defined as non-susceptibility to at least one agent in all but two or fewer antimicrobial categories (i.e., bacterial isolates remain susceptible to only one or two antimicrobial categories). Pandrug resistance (PDR) was defined as non-susceptibility to all agents in all antimicrobial categories. [11]

The study was approved by institutional ethics committee and consent of guardians was taken before including the children into the study.

\section{Statistical Analysis:}

Descriptive analysis was done by calculating frequencies, mean values and percentages. Statistical analysis was done using SPSS for windows version 22.0. Chi-square test used for the comparison of categorical variables. P-value less than 0.05 were considered statistically significant.

\section{Results}

A total of 130 children (250 episodes of febrile neutropenia) were included in this study. Male to female ratio was 1.7: 1. Mean age at presentation was 6.5 years (range: 6 months -15 years, standard deviation: 4.29 years). Mean duration of fever was 72 hours (range: 2- 20 days, standard deviation: 3.09 days). Acute Lymphoblastic Leukaemia (ALL) was the most common malignancy noted at 186 episodes (74\%), followed by Acute Myeloid Leukaemia (AML) at 47 episodes (19\%) followed by Lymphoma, Ewing's sarcoma and rhabdomyosarcoma. Respiratory symptoms (13\%) were the most common presentation, followed by musculoskeletal symptoms ( $11 \%)$. Nine percent of the episodes had features of shock and were admitted to paediatric intensive care unit. No obvious focus of infection was noted in $57 \%$ 
episodes. During the course of hospitalization all the episodes had ANC $<500$; ANC $<200$ was observed in $50 \%$ of the episodes. The average number of cultures sent per episode of neutropenia was 1.536. A total of 384 cultures were sent, of which $92(24 \%)$ were positive. Blood cultures constituted 246 of these of which 65 were positive. Once the blood cultures were sent, empiric antibiotics were started. In case of no clinical improvement/ worsening, further culture samples were taken under strict asepsis. A total of 92 urine culture, 26 endotracheal trap aspirate cultures and 20 cerebrospinal fluid cultures were obtained. Most common organisms isolated were gram negative bacteria (48 isolates, $52.2 \%$ ), followed by gram positive cocci (33 isolates, $35.8 \%$ ), fungi ( 6 isolates, $6.5 \%$ ) and 5 polymicrobial cultures (5.5\%) in 92 culture positive episodes. [Figure 1] Amongst the gram negative bacteria, the lactose fermenters (29 isolates, 31.5\%) predominated with Escherichia coli being the most common (19 isolates, 20.6\%) followed by Klebsiella (10 isolates, 10.8\%). Non fermenting gram negative bacteria constituted 19 isolates, (20.6\%) including Pseudomonas ( 6 isolates, $6.5 \%$ ). Amongst gram positive coagulase negative staphylococcus (CONS) was the most common (29\%), followed by Streptococcus and Enterococcus.

The sensitivity patterns have been displayed in tables. [Table 1,2] We noticed that almost all gram positive isolates in our cultures showed excellent response to first and second line antibiotics with CONS showing sensitivity of $96 \%$ to amikacin, $100 \%$ to ceftazidime, piperacillin-tazobactam, meropenem, vancomycin and colistin. Streptococci and Enterococci had less susceptibility to first line antibiotics but responded well to vancomycin and meropenem. The gram negative bacteria on the other hand had poor response to ceftazidime and relatively better response to amikacin. Lactose fermenting bacteria like Escherichia coli had sensitivity to ceftazidime in only $36 \%$ and NFGNB was sensitive in $25 \%$. Escherichia coli strain was sensitive to amikacin in $71 \%$ episodes and Pseudomonas had excellent susceptibility (100\%) as well. Klebsiella and NFGNB (other than Pseudomonas) had relatively poor sensitivity to amikacin. All gram negative bacteria both lactose-fermenters and non-fermenters showed a mean sensitivity of only 16.7, 24.7 and 33.7 to piperacillin-tazobactom, ceftazidime and meropenem respectively.. NFGNB (other than Pseudomonas) and Klebsiella were $44 \%$ sensitive to amikacin. Pseudomonas and Escherichia coli had a sensitivity of $100 \%$ and $71 \%$ respectively to amikacin. The mean of sensitivity of all gram negative bacteria to amikacin was 57. NFGNB (other than Pseudomonas) had a sensitivity of $68 \%$ to colistin. Escherichia coli and Klebsiella showed even higher sensitivity of $90 \%$, $80 \%$ respectively to colistin.

First line antibiotics (ceftazidime and amikacin) were started for all the episodes, however, 78 episodes required second line antibiotics. These 78 episodes comprised 47 positive cultures (out of 92 positive cultures) and 12 deaths (out of 13 deaths). Of the 47 positive cultures within this group 31 belonged to gram negative group, while only 9 belonged to gram positive group. Episodes with gram negative isolates were more likely to require second line antibiotics (51\%). [Table 3] Out of the 12 deaths in this group eight died due to gram negative bacteraemia. [Figure 2]

Pan drug resistance was found in 3 gram negative cultures, associated with 2 deaths. Extreme drug resistance was seen in 20 gram negative isolates, associated with 5 deaths. Multi drug resistance was seen in 13 gram negative isolates, associated with 1 death. Most drug resistance (i.e., MDR, XDR and 
PDR, a total of 36) was noticed in the gram negative group. [Table 4] Escherichia coli and Klebsiella were found to have more resistant isolates and highest mortality. [Figure 3]

Much higher mortality was seen in episodes with gram negative isolates and especially higher in drug resistant isolates. [Table 5] Amongst those requiring second line antibiotics, 12 deaths were reported of which eight deaths were due to gram negatives, one death was due to gram positive and two deaths due to fungal infection. In the group that required only first line antibiotic, most common isolate was gram positive bacteria (24 isolates) while gram negative bacteria relatively lesser (17 isolates). Only one death was reported in this group. There was only one death due to viral cause which was due to dengue haemorrhagic fever.

\section{Discussion}

The data from western countries show a prevalence of gram positive organisms. [12,13] However, in developing countries like India, there has been a rising trend of gram negative bacteremia. [14] In our study the gram negative bacteria accounted for $52 \%$ of the episodes. Our study reinforces this recent trend and demonstrates that the switch to gram positive bacteria amongst the western countries may be just limited to the developed countries. [16] Cattaneo et al also stated an evidence of epidemiological shift from gram positive to gram negative bacteria in febrile neutropenic patients with Escherichia coli being the most frequent organism. [17] There have been other reports of emergence of gram negative bacteria, predominantly Pseudomonas and Enterobacteriaceae. [18]

Since febrile neutropenia is a clinical emergency which needs to be addressed timely, empiric antibiotics are administered as soon as possible, even before culture sensitivity results are available, which may take 2-3 days. A delay of more than 48 hours in the administration of appropriate antibiotics may result in a mortality rate as high as $50 \%$. [19] In previous studies mortality has been variably reported from $5 \%$ to $39 \%$ in other developing countries. $[20,21]$ The overall mortality in our study was $13(10 \%$ of the total patients with febrile neutropenia). The interesting fact we noted was that $61.5 \%$ of the deaths were due to gram negative bacteremias.

In our study, initial empirical antimicrobial treatment was found to be appropriate in only $35 \%$ cases, probably due to lower susceptibility to ceftazidime. Due to higher load of drug resistant (MDR, PDR, XDR) bacteria, febrile neutropenic patients with gram negative bacteremia usually present with rapid disease onset and multiple complications. Similar presentations were also noticed by $\mathrm{K}$ Govind Babu et al in a study conducted in southern India. [22] The sick patients required second line antibiotics. This group also had significantly more deaths ( $61 \%$ of all deaths) as well as more PICU admissions. All patients who died had required PICU admission.

Irfan $\mathrm{S}$ et al had described emergence of carbepenem resistant extended spectrum beta lactamases. Similar to this study we also had a predominance of piperacillin-tazobactam and meropenem resistant extended spectrum beta lactamase producing organisms. Emergence of carbepenem resistant extended spectrum beta lactamase organisms have also been reported by Micozzi et al. [23] Few recent studies in 
China have also reported increasing requirement of higher antibiotics (almost 70\%) in neutropenic patients, similar to our study, wherein a total of 78 episodes (47 out of 92 culture positives) required escalation i.e., in nearly $51 \%$ cases, first line antibiotics did not suffice. A significant proportion in this group was GNB which constituted $31(66 \%)$ of these cases.

Three out of the total GNB that required second line antimicrobials were pan drug resistant (resistant to all drugs). While 20 were classified to be extremely drug resistant. Eight out of the total 13 deaths were caused by these highly resistant bacteria. Hence, the importance of knowing the locally prevalent pathogens and their susceptibility pattern cannot be emphasized enough.

We found a higher prevalence of carbepenem resistant extended spectrum beta lactamase producing organisms with poor sensitivity to first and second line antibiotics in a substantial number of patients in our hospital set up. Although relatively good sensitivity was noted with amikacin, monotherapy with amikacin alone will not suffice and it is advisable to add higher antibiotics like colistin earlier, in case of clinical worsening.

Epidemiology and resistance patterns in local hospitals should be considered when choosing empirical antibiotic treatments as the optimal treatment strategy might vary in different local settings.

Since most of the oncology units, even in developing countries now have Bactec automated culture systems which can detect the involved organisms sooner, we propose that early escalation to higher antibiotics be promoted, based on local culture sensitivity pattern, especially in patients with gram negative bacteremia and clinical deterioration.

\section{Conclusions}

Emergence of drug resistant gram negative microbes is a concern which has to be addressed in order to reduce the mortality and morbidity associated with febrile neutropenia. Gram negative bacteraemia is more common and the leading cause for deaths in pediatric oncology department especially in developing countries. Aminoglycosides may need to be included for upfront empiric antibiotic coverage for children with febrile neutropenia especially in the context of drug resistance in the developing world. Since monotherapy won't be able to cover all gram negative strains, broad spectrum antibiotics based on local culture sensitivity profiles should be started.

In view of higher prevalence of gram negative isolates and emergence of multi drug resistance, frequent audits of resistance patterns should guide choice of antimicrobials in febrile neutropenia management. Our results show the importance of surveillance, monitoring resistance frequencies and identifying risk factors specific to each region. Early administration of restricted antibiotics may need to be considered early if automated culture systems reveal gram negative bacteremia especially if patients are unstable. (earliest detection of the organism) to prevent complications like sepsis and multi organ failure.

\section{Abbreviatons}


ALL

AML

ANC

CONS

GNB

GPB

ICU

MDR

NFGNB

PDR

UTI

XDR

\section{Declarations}

\section{ETHICS APPROVAL:}

We do hereby declare that this study has been approved by St. John's Medical College and Hospital Institutional Review Board vide ref. no. 3/13-11(1)2011.

CONSENT FOR PUBLICATION:

Not applicable

\section{AVAILABILITY OF DATA AND MATERIALS :}

All data generated or analysed during the study is included in this published article and in supplementary files.

\section{COMPETING INTERESTS:}

The authors declare that they have no competing interests.

\section{FUNDING:}

The authors declare that they have no funding resources for this study. 
VJ, AP and KM conceived and planned the study. VJ and TD collected the data. VJ performed statistical analysis and generated results of the study. VJ, AP and KM performed literature review and drafted the manuscript. AP contributed in provision of clinical information and approved the final manuscript. All authors read and approved the final manuscript.

\section{ACKNOWLEDGEMENT:}

Not applicable.

\section{References}

1. Alp S, Akova M. Management of febrile neutropenia in the era of bacterial resistance. Ther Adv Infect Dis. 2013:1(1): 37-43.

2. Klastersky, J. de Naurois, K. Rolston, B. Rapoport, G. Maschmeyer, M. Aapro and J. Herrstedt. Management of Febrile Neutropaenia: ESMO Clinical Practice Guidelines. Ann Oncol (2016) 27 (suppl 5): v111-v118.

3. Gajdacs M, Burian K, Terhes G. Resistance levels and epidemiology of non-fermenting gram-negative bacteria in urinary tract infections of inpatients and outpatient (RENFUTI): a 10-year epidemiological snapshot. Antibiotics (Basel). 2019;8:3.

4. Gajdacs M, Urban E. Epidemiological trends and resistance associated with stenotrophomonas maltophilia bacteremia: a 10-year retrospective cohort study in a tertiary-care hospital in hungary. Diseases. 2019;7(2):41.

5. Gajdacs M. The continuing threat of methicillin-resistant staphylococcus aureus. Antibiotics (Basel). 2019;8:2.

6. Klastersky J, Ameye L, Maertens $\mathrm{J}$, et al. Bacteraemia in febrile neutropenic cancer patients. Int $\mathrm{J}$ Antimicrob Agents. 2007;30(Suppl 1):S51-S59.

7. Siddaiahgari S, Manikyam A, Kumar KA, Rauthan A, Ayyar R. Spectrum of systemic bacterial infections during febrile neutropenia in pediatric oncology patients in tertiary care pediatric center. Indian J Cancer 2014;51:403-5.

8. Singer M, Deutschman CS, Seymour CW, et al. The third international consensus definitions for sepsis and septic shock (Sepsis-3). JAMA. 2016 Feb 23;315(8):801-810.

9. Seymour CW, Liu VX, Iwashyna TJ, et al. Assessment of clinical criteria for sepsis: for the third international consensus definition for sepsis and septic shock (Sepsis-3). JAMA. 2016 Feb 23;315(8):762-774.

10. World Health Organization. Geneva, Switzerland: WHO. Dengue: Guidelines for Diagnosis, Treatment, Prevention and Control, 2009. 
11. P. Magiorakos, A. Srinivasan, R.B. Carey, Y. Carmeli, M.E. Falagas et al. Multi-drug resistant, Extensively-drug resistant and Pan-drug resistant bacteria: An international expert proposal for interim standard definition for acquired resistance. Clin Microbiol Infect 2012;18: 268-81.

12. Meidani M, Bagheri A, Khorvash F. A Populaton- Based Study of Bacterial Spectrum in Febrile neutropenic Patients, Jundishapur J Microbiology. 2013:6(2): 150- 6.

13. Gonzalez-Barca E, Fernandez-Sevilla A, Carratala J, Granena A, Gudiol F. Prospective study of 288 episodes of bacteremia in neutropenic cancer patients in a single institution. Eur $\mathrm{J}$ Clin Microbiol Infect Dis. 1996: 15(4): 291-6

14. Rose W, Veeraraghavan B, George B. Bloodstream infections in children with febrile neutropenia: Isolates and their antimicrobial susceptibility profile. Indian J Cancer 2015;52:495-6

15. Irfan S, Faiza Idrees, Vikram Mehraj,Faizah Habib, Salman Adil and Rumina Hasan Emergence of Carbapenem resistant Gram negative and vancomycin resistant Gram positive organisms in bacteremic isolates of febrile neutropenic patients: A descriptive study BMC Infectious Diseases20088:80.

16. Greer J.P., Arber D.A., Glader B. Wintrobe's Clinical Hematology. $13^{\text {th }}$ William and Wilkins; Baltimore, Md:2013.pp.1836/188.

17. Cattaneo C, Quaresmini G, Casari S, Capucci MA, Micheletti M, Borlenghi E, et al. Recent changes in bacterial epidemiology and the emergence of fluoroquinolone- resistant Escherichia coli among patients with haematological malignancies: results of a prospective study on 823 patients at a single institution. J Antimicrob Chemother. 2008: 61(3):721-8.

18. Klastersky J, Ameye L, Maertens J, Georgala A, Muanza F, Aoun M. Bacteremia in febrile neutropenic cancer patients. Int J Antimicrob Agents. 2007: 30-9

19. Naeem A., Hussain Y., Ahmed B., Aziz M.T. et al. A prospective study of cefepime versus Ticarcillin/clavulanate as empiric treatment of febrile neutropenia in lymphoma patients. J. Pakistan Med Assoc. 2011;61(1):18-22.

20. B. Karanwal, B.J. Parikh, P. Goswami, H.P. Panchal, B.B. Parekh, K. B. Patel. Review of clinical profile and bacterial spectrum and sensitivity patterns of pathogens in febrile neutropenic patients in hematological malignancies: a retrospective analysis from a single center. Indian Journal of Medical and Paediatric Oncology, Vol. 34, No. 2, 2013, pp85-88.

21. S. Lima, M.S. Franca, G.H. Martinho, L. A. Jesus, R.M.C. Romanelli et al. Neutropenic patients and their infectious complications at a university hospital. Revista Brasileira de Hematologia e Hemoterapia, vol. 35, no. 1 2013, pp 18-22

22. Govind Babu, D. Lokanatha, K.C. Lakshmaiah, M.C. Suresh Babu, Linu A. Jacob et al. Indian J Med Paediatr Oncol. 2016. 37 (3): 174-182.

23. Micozzi A, Gentile G, Minotti $C$ et al. Carbepnem resistant Klebsiella pneumoniae in high risk haematological patients; factors favouring spread, risk factors and outcome of Carbepenem resistant Klebsiella pneumoniae bacteremias. BMC Infect Dis. 2017;17(1):203. 


\section{Tables}

Table1: Sensitivity pattern of gram positive organisms.

\begin{tabular}{|c|c|c|c|c|c|c|c|c|}
\hline Organisms & $\begin{array}{l}\text { Number } \\
\text { (\% out of total } \\
\text { culture positive) }\end{array}$ & $\begin{array}{l}\text { Ceftazi- } \\
\text { dime } \\
\text { Sensi- } \\
\text { tivity }\end{array}$ & $\begin{array}{l}\text { Amika- } \\
\text { cin } \\
\text { Sensi- } \\
\text { tivity }\end{array}$ & $\begin{array}{l}\text { Piperacillin } \\
\text { Tazobactam } \\
\text { Sensi- } \\
\text {-tivit }\end{array}$ & $\begin{array}{l}\text { Meropenem } \\
\text { Sensi- } \\
\text { tivity }\end{array}$ & $\begin{array}{l}\text { Colistin } \\
\text { Sensi- } \\
\text { tivity }\end{array}$ & $\begin{array}{l}\text { Tigecycline } \\
\text { Sensi- } \\
\text { tivity }\end{array}$ & $\begin{array}{l}\text { Vancomycin } \\
\text { Sensi-tivity }\end{array}$ \\
\hline CONS & $27(29)$ & $100 \%$ & $96 \%$ & $100 \%$ & $100 \%$ & $100 \%$ & $83 \%$ & $100 \%$ \\
\hline Staph. Aureus & $1(1)$ & 0 & $100 \%$ & $100 \%$ & $100 \%$ & $100 \%$ & $100 \%$ & $100 \%$ \\
\hline Streptococcus & $2(2)$ & $50 \%$ & $33 \%$ & $50 \%$ & $100 \%$ & $50 \%$ & $50 \%$ & $100 \%$ \\
\hline Enterococcus & $3(3.2)$ & 0 & 0 & 0 & 0 & 0 & 0 & $100 \%$ \\
\hline
\end{tabular}

Table2: Sensitivity pattern of gram negative organisms. 


\begin{tabular}{|c|c|c|c|c|c|c|c|}
\hline Organisms & $\begin{array}{l}\text { Number } \\
\text { (\% out of total culture } \\
\text { positive) }\end{array}$ & $\begin{array}{l}\text { Ceftazidime } \\
\text { Sensitivity } \\
\text { (\%) }\end{array}$ & $\begin{array}{l}\text { Amikacin } \\
\text { Sensi- } \\
\text { tivity }\end{array}$ & $\begin{array}{l}\text { Piperacillin- } \\
\text { Tazobactam } \\
\text { Sensitivity }\end{array}$ & $\begin{array}{l}\text { Meropenem } \\
\text { Sensi-tivity }\end{array}$ & $\begin{array}{l}\text { Colistin } \\
\text { Sensi- } \\
\text { tivity }\end{array}$ & $\begin{array}{l}\text { Tigecycline } \\
\text { Sensi-tivity }\end{array}$ \\
\hline $\begin{array}{l}\text { NFGNB } \\
\text { (except } \\
\text { pseudomonas) }\end{array}$ & $13(14)$ & 25 & $44 \%$ & $20 \%$ & $29 \%$ & $68 \%$ & $43 \%$ \\
\hline Pseudomonas & $6(6.5)$ & 25 & $100 \%$ & 0 & $20 \%$ & 0 & 0 \\
\hline E.coli & 19 (20.6) & 36 & $71 \%$ & $21 \%$ & $43 \%$ & $90 \%$ & $50 \%$ \\
\hline Klebsiella & $10(11)$ & 13 & $44 \%$ & $22 \%$ & $44 \%$ & $80 \%$ & 0 \\
\hline $\begin{array}{l}\text { Mean of } \\
\text { sensitivity }\end{array}$ & & 24.7 & 57 & 16.7 & 33.7 & 65 & 29 \\
\hline
\end{tabular}

Table3: Need for second line antibiotics for Gram negative Bacteria versus others

\begin{tabular}{|l|l|l|}
\hline & Use of second line antibiotics & Use of first line antibiotics \\
\hline Gram negative bacteria & 31 & 17 \\
\hline Others & 16 & 28 \\
\hline
\end{tabular}

*significant at the level of $p<0.05$

Table4: Drug Resistance (MDR, XDR and PDR) in gram negative bacteria versus others

\begin{tabular}{|l|l|l|}
\hline & Sensitive & Resistance \\
\hline Gram negative bacteria & 12 & 36 \\
\hline Gram positive bacteria & 27 & 06 \\
\hline
\end{tabular}

*significant at the level of $p<0.05$.

Table5: Mortality in gram negative bacteria versus others

\begin{tabular}{|l|l|r|}
\hline & Deaths & Alive \\
\hline Gram negative bacteria & 8 & 23 \\
\hline Others & 4 & 41 \\
\hline
\end{tabular}




\section{Figures}

\section{Number of positive cultures}

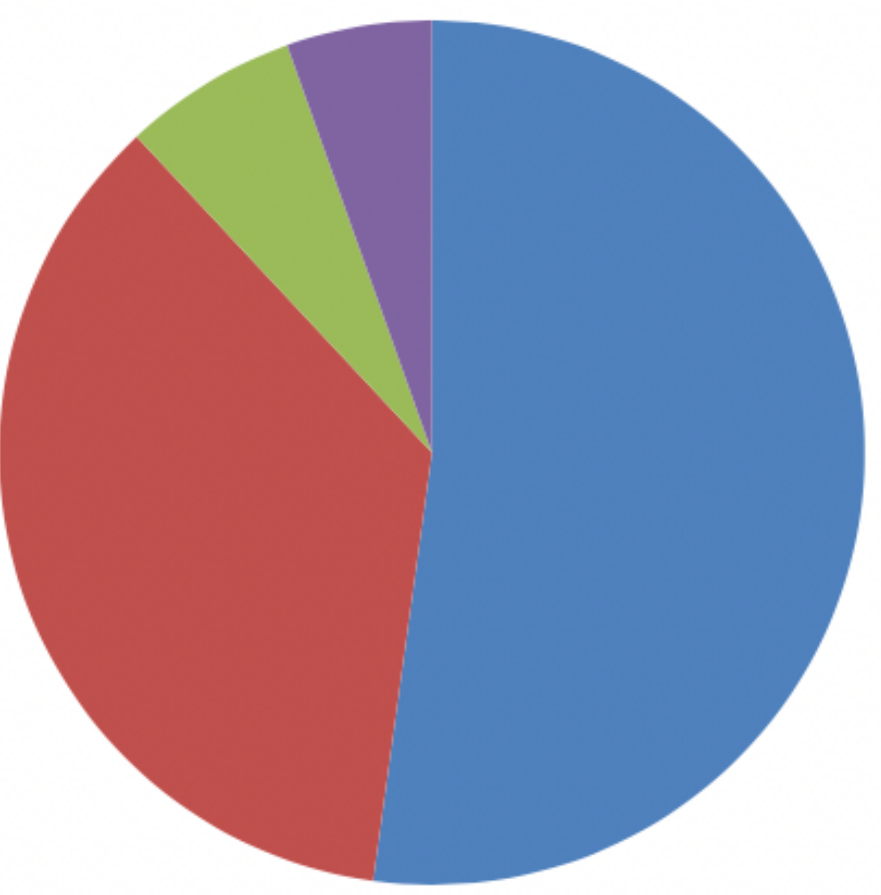

- Gram negative bacteria (52.1\%)

- Gram positive bacteria (35.8\%)

Fungus (6.5\%)

- Polymicrobial (5.4\%)

Figure 1

Gram staining pattern 


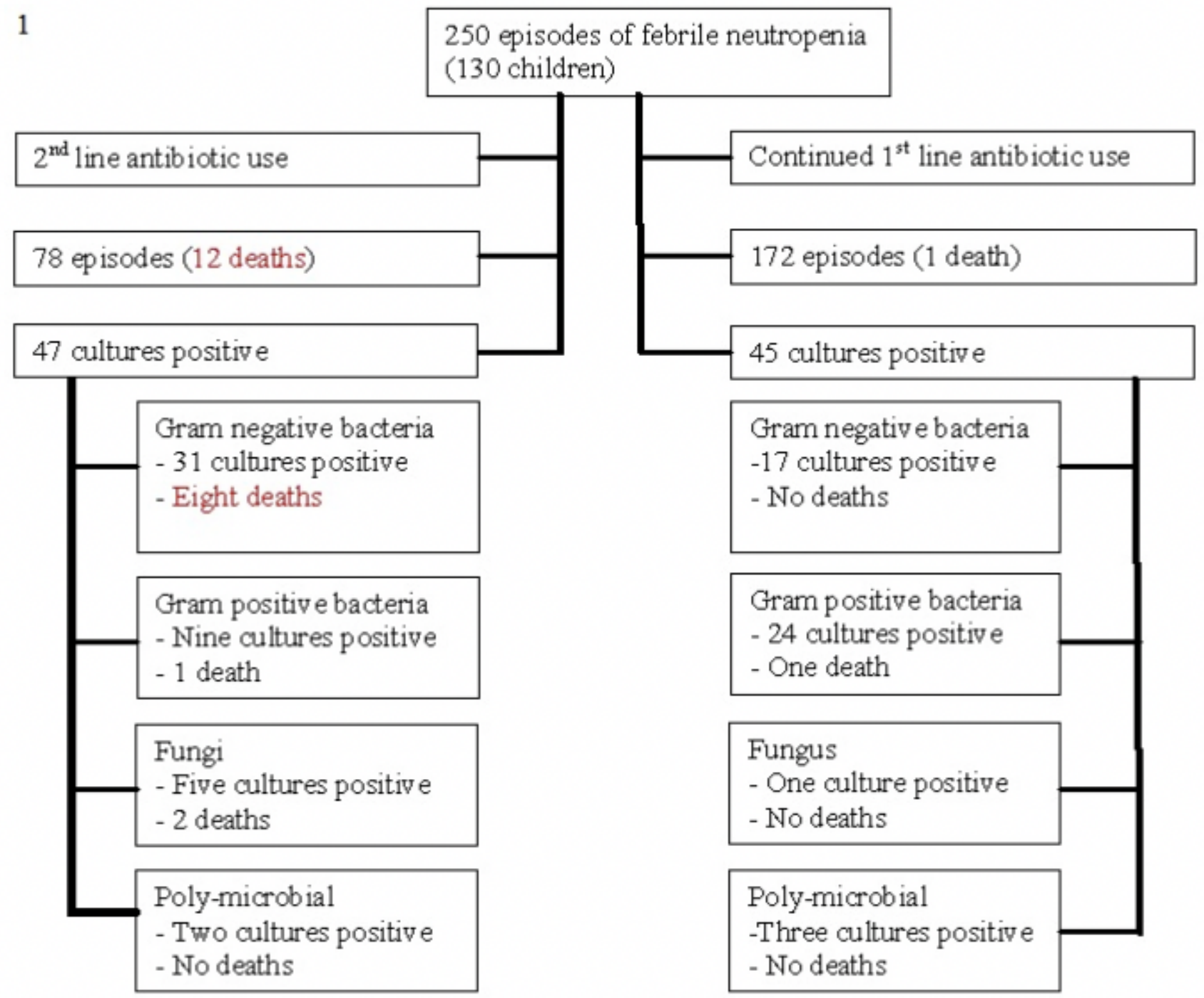

Figure 2

Breakdown of two fifty episode based on culture sensitivity 


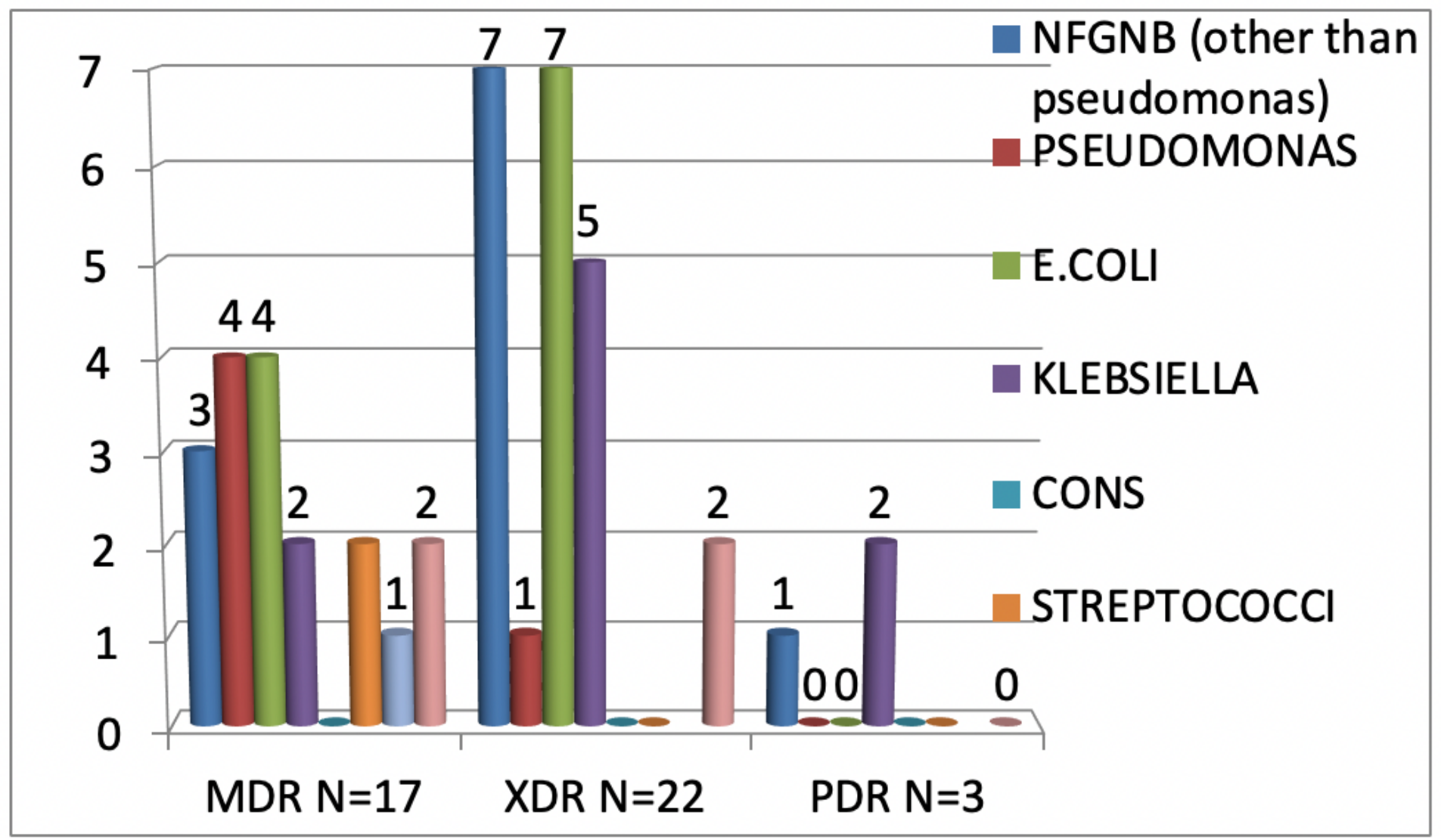

Figure 3

Common drug resistant organisms. 